\title{
ASPECTOS HISTÓRICOS DA PRESSÃO ARTERIAL DE OXIGÊNIO E ESPIROMETRIA RELACIONADOS À OPERAÇÃO ABDOMINAL
}

\author{
Historical aspects of arterial oxygen pressure and spirometry related to abdominal \\ operations
}

\author{
Gilson Cassem RAMOS, Edísio PEREIRA, Salustiano GABRIEL-NETO, Enio Chaves de OLIVEIRA
}

ABCDDV/639

Ramos GC, Pereira E, Gabriel-Neto S, Oliveira EC. Aspectos históricos da pressão arterial de oxigênio e espirometria relacionados à operação abdominal ABCD Arq Bras Cir Dig 2009;22(1):50-6

RESUMO - Introdução - Operações, principalmente de abdome superior, cursam no pós-operatório com hipoxemia e distúrbios ventilatórios restritivos. O objetivos da presente revisão foram: a) fazer retrospecto histórico das técnicas espirométricas e da avaliação laboratorial da hipoxemia, por meio da pressão arterial de oxigênio $(\mathrm{PaO} 2)$ e, b) rever os principais fatores responsáveis pelas alterações da função pulmonar pós-operatória. Métodos - Fez-se revisão histórica sobre os principais aspectos espirométricos e da medida da $\mathrm{PaO}$ 2, correlacionando esses exames como aferidores da função pulmonar, após operações abdominais. Conclusões - Operações em andar superior do abdome podem cursar com hipoxemia e distúrbios ventilatórios restritivos, cuja principal causa é a disfunção diafragmática, que pode ser minimizada por meio de laparoscopia e um eficaz tratamento da dor pós-operatória.

DESCRITORES - Operações abdominais. Função pulmonar. Pós-operatório.

\section{INTRODUÇÃO}

Procedimentos cirúrgicos tendem a promover hipoxemia tardia ${ }^{70}$ e distúrbios ventilatórios restritivos pósoperatórios, especialmente após operações abdominais ${ }^{2}$. Dessa maneira, o maior comprometimento é observado depois de operações de andar superior do abdome, seguido de operações torácicas, vindo a seguir procedimentos de abdome inferior ${ }^{1}$. Assim, os pulmões tornam-se os mais importantes órgãos-alvos relacionados à morbi-mortalidade pós-operatória ${ }^{1}$.

O objetivo do presente estudo é fazer retrospecto histórico da espirometria e da pressão arterial de oxigênio ( $\mathrm{PaO} 2)$, e da relação dessas variáveis com operações abdominais. Por outro lado determinar as causas das disfunções pulmonares pós-operatórias e formas de minimizá-las, o que pode reduzir complicações pulmonares pós-operatórias.

\section{MÉTODO}

Foram utilizadas a bases de dados do Pubmed (www. ncbi.nlm.nih.gov/pubmed/) para revisão da literatura internacional (palavras-chave: postoperative pulmonary complications, pulmonary function, blood gas, spirometry) e

Trabalho realizado no Programa de Pós-graduação em Ciências da Saúde da Universidade de Brasília - UnB, Brasília, DF, Brasil.

Endereço para correspondência: Gilson Cassem Ramos, e-mail: gilson.ramos@terra.com.br do Lilacs (www.bireme.br) (palavras-chave: complicações pulmonares pós-operatórias, gases sanguineos, espirometria, função pulmonar) para revisão da literatura médica nacional/latino americana. Alguns artigos históricos foram selecionados a partir de citações da literatura levantada.

\section{Espirometria}

É a prova de função pulmonar de mais simples execução e a mais importante na prática clínica. É obtida por um espirômetro, por meio do qual pode-se determinar volumes (estáticos e dinâmicos) e capacidades pulmonares (soma de dois ou mais volumes). Utilizando registro gráfico, obtém-se uma curva de volume/tempo, sendo possíveis cálculos de volumes e fluxos instantâneos ${ }^{14}$. Completando as informações do exame, os espirômetros computadorizados, por derivação matemática, geram curvas de fluxo/ volume. Assim, existem espirômetros comercialmente disponíveis que são capazes de gerar mais de 23 variáveis espirométricas, contudo com os parâmetros capacidade vital forçada (CVF), volume expiratório forçado em um segundo (VEF1) e o índice de Tiffeneau (VEF1/CVF) diagnostica-se a grande maioria dos distúrbios ventilatórios. O VEF1 é a medida de função pulmonar mais útil clinicamente ${ }^{9}$, ao passo que a CVF é o teste de função pulmonar mais importante ${ }^{56}$. Valores normais de cada uma destas variáveis baseiam-se na idade, gênero, peso e altura do indivíduo. Dessa forma, o espirômetro emite os valores normais (preditos) dos parâmetros espirométricos, obedecendo critérios teóricos pré-estabelecidos, após o equipamento ser alimentado com os dados individuais do indivíduo. Assim, o limite inferior da normalidade para 
a CVF e o VEF1 é considerado $80 \%$ dos valores teóricos preditos pelo espirômetro. Já para relação VEF1/CVF é de aproximadamente $70 \%$ dos valores teóricos ${ }^{33,57}$. Os distúrbios ventilatórios são classificados em obstrutivos, restritivos e mistos. Isoladamente, a redução da razão VEF1/ CVF faz o diagnóstico certeza de obstrução, cujas causas mais frequentes são: asma, enfisema e bronquite crônica. Já os distúrbios restritivos são caracterizados pela queda da CVF, com o índice de Tiffeneau normal. As suas principais causas são: pneumonias, operação abdominal e torácica, fibroses pulmonares, derrames pleurais, tumor de parede torácica e obesidade. Os distúrbios mistos são mais difíceis de se caracterizar e ocorrem pela sobreposição dos achados espirométricos de obstrução com restrição (diminuição da CVF e da relação VEF1/CVF). Algumas de suas causas são: doenças granulomatosas e associação de doenças obstrutivas com restritivas (bronquite por tabagismo + silicose; enfisema + sequela de tuberculose pulmonar). $\mathrm{O}$ distúrbio ventilatório, de acordo com sua gravidade (Tabela 1), pode ser classificado em grau leve, moderado e grave ou acentuado, segundo os valores da CVF e do VEF $1^{68}$.

TABELA 1 - Gravidade dos distúrbios ventilatórios ${ }^{68}$.

\begin{tabular}{llll}
\hline Grau & VEF1(\%) & CVF (\%) & VEF1/CVF \\
\hline Leve & $60-$ LI & $60-$ LI & $60-$ LI \\
Moderado & $41-59$ & $51-59$ & $41-59$ \\
Grave & $\leq 40$ & $\leq 50$ & $\leq 40$ \\
\hline
\end{tabular}

LI = Limite inferior da normalidade (valores pré-calculados pelo espirômetro): aproximadamente $80 \%$ para VEF1 e CVF e $70 \%$ para VEF1/CVF.

De acordo com os sistemas, os espirômetros são classificados em abertos ou fechados. Nos abertos, a inspiração máxima é executada fora do sistema, para depois a peça bucal ser utilizada para a expiração. Nos fechados, ambas as manobras são realizadas no equipamento. Segundo os métodos de medição de volume, são classificados em diretos, cujo volume de ar mobilizável é medido diretamente durante o teste, e indiretos, quando se avalia o volume pulmonar por meio do fluxo captado e integrado eletronicamente ${ }^{22}$. Os diretos são subdivididos em espirômetros do tipo selados em água, de pistão e de fole. Os indiretos são de quatro tipos: sensores de fluxo de pressão diferencial, termistores, turbinômetros e sensores de fluxo ultra-sônicos ${ }^{62}$. As principais vantagens dos espirômetros de fluxo são o seu menor tamanho, permitindo aparelhos portáteis; a facilidade de serem higienizados; e, como medem o fluxo diretamente, teem a vantagem de medir todos os fluxos com maior precisão. Sua principal desvantagem é relacionada a erro na presença de fluxo zero, que pode conduzir à distorção de volume, especialmente no cálculo da CVF. No final do decênio de 1980 e início da década de 1990 introduziu-se na prática clínica, a utilização de espirômetros portáteis. Tratase de aparelhos menores, baseados em sensores de fluxo, de fácil manuseio e locomoção e, o mais importante, precisos nas aferições dos diferentes parâmetros espirométricos ${ }^{44}$. Com o seu advento, o teste passou a alcançar, mais facil- mente, pacientes com dificuldade de serem transportados a um laboratório de espirometria, tais como pacientes em convalescença de operações. Assim, diagnóstico e pesquisa em espirometria, nesses pacientes, tornaram-se mais fáceis, podendo o exame ser feito, inclusive, à beira do leito do enfermo. Durante a realização da espirometria, realiza-se a prova broncodilatadora. Ela é parte integrante do teste espirométrico e compara as duas fases do exame: a basal, pré-broncodilatador e a outra, pós-broncodilatador. Sua indicação habitual é identificar e avaliar o componente espástico na gênese do processo obstrutivo. Utiliza-se um agente beta adrenérgico de ação rápida, normalmente o salbutamol ou salbuterol, pela via inalatória. Administram-se quatro jatos de $100 \mu \mathrm{g}$ do fármaco, por câmara de expansão $^{72}$. Aguardam-se de 15 a 20 minutos e realiza-se nova medida, que é avaliada usualmente por variações do VEF1 e CVF. As principais indicações de espirometria são $0^{55}$ : a) investigação de dispnéia; b) qualificação e quantificação dos distúrbios respiratórios; c) avaliação da eficácia de terapêutica medicamentosa nas doenças respiratórias; d) monitorar trabalhadores com risco de doenças respiratórias ocupacionais; e) reabilitação cardiopulmonar; f) avaliação de risco cirúrgico; e g) em medicina esportiva. As contraindicações para o teste são relativas e estão relacionadas a algumas situações clínicas que podem se transformar em risco para o paciente durante as manobras espirométricas. Podem-se enumerar: hemoptise de causa desconhecida, pneumotórax, instabilidade cardiocirculatória, infarto do miocárdio ou tromboembolismo pulmonar recentes, aneurismas (torácico, abdominal ou cerebral), operação ocular recente e náuseas ou vômitos ${ }^{64}$.

\section{Aspectos históricos da espirometria}

Os primeiros relatos científicos a respeito dos volumes pulmonares são do início do século XIX. Mais precisamente, em 1800, Davy descreveu como ar residual, modernamente chamado de volume residual, o volume de ar que permanece nos pulmões após expiração máxima (citado por Christie, 1932) ${ }^{10}$. O inglês Hutchinson $(1846)^{27}$, médicocirurgião, criou o espirômetro. $\mathrm{O}$ modelo desenvolvido por ele consistia em uma campânula flutuando em tanque com água. O pesquisador relatou a observação de mais de 2000 indivíduos e descreveu a capacidade vital como sendo o volume de ar que pode ser expirado a partir de uma inspiração máxima até sforço expiratório também máximo (conceito atual de CVF). Ele correlacionou a redução desse parâmetro respiratório com maior morbi-mortalidade precoce e ainda descreveu o volume pulmonar total ou capacidade total, atualmente chamada de capacidade pulmonar total, como a soma do ar residual com a capacidade vital. Outros ${ }^{39}$ pesquisadores descreveram como ar residual funcional, indicado também como capacidade média ou normal, aquele volume de ar que permanece nos pulmões após expiração normal; parâmetro referido nos dias de hoje como capacidade residual funcional (CRF). Nessa mesma época, foram conceituados os termos ar complementar e ar de reserva (ou ar suplementar). O primeiro refere-se ao volume máximo de ar que pode ser inspirado, 
partindo-se do nível expiratório de repouso. É indicado, atualmente, como capacidade inspiratória. O segundo é relativo ao volume de ar máximo que pode ser expirado a partir do nível final da expiração normal. É apontado, nos dias atuais, como volume de reserva expiratório. A quantidade máxima de ar, que pode ser inspirada a partir da posição final da inspiração normal, era mencionada como ar complementar menos ar corrente e, atualmente, volume de reserva inspiratório. Ar corrente, hoje reportado como volume corrente, foi definido como sendo o volume de ar expirado em cada respiração normal. Davy, em 1800 (citado por Van Slyke, 1923) ) $^{75}$ mediu o volume residual dos seus próprios pulmões inalando uma mistura de hidrogênio. Outros autores (citado por Van Slyke, 1923) ${ }^{75}$ utilizando o mesmo princípio de Davy, mensurou a CRF e o volume do espaço morto. Ambos pesquisadores aplicaram o princípio da respiração forçada, que, anos mais tarde, foi substituído pelo método da diluição prolongada ${ }^{75}$. Anos mais tarde, foi instituido um outro método (diluição de $\mathrm{O} 2$ sem respiração forçada $)^{10}$ para aferir a CRF e proposta terminologia única para os parâmetros pulmonares volumétricos, haja vista a diversidade de nomes oferecidos a um mesmo volume pulmonar, o que gerava confusão. No entanto, padronização somente foi conseguida quando um grupo de fisiologistas americanos, sob o comando de Pappenheimer $(1950)^{51}$, reuniram-se e adotaram a terminologia atual, mundialmente aceita. Nas décadas de 1940 e 1950, foram desenvolvidos novos métodos para medir os volumes estáticos dos pulmões, utilizados atualmente. São eles: diluição do gás hélio, que substituiu o hidrogênio de Van Slike; circuito aberto com nitrogênio e pletismografia ${ }^{79}$. Os mais relevantes estudos sobre fisiopatologia pulmonar só ocorreram a partir da década de 1940 . Nesse período, o espirômetro foi acoplado a um quimógrafo, o que permitiu correlacionar as variações do volume de ar inspirado ou expirado com variações do tempo. Um grande avanço foi dado, em Paris, por Tiffeneau e Pinelli ${ }^{73}$, que anunciaram a medida do VEF1. Nos EUA, Gaensler, aproximadamente quatro anos mais tarde, descreveu a mesma variável, porém a referindo como "tempo de capacidade vital" 20 . Assim, os pioneiros da espirometria cronometrada foram Tiffeneau e Gaensler. No final dos anos do decênio de 1950, foram introduzidos os estudos a respeito de curva fluxo/volume ${ }^{28}$. Na evolução dos espirômetros advieram outras importantes modificações. O retentor de $\mathrm{CO} 2$ (um recipiente com cal sodada) foi acoplado ao equipamento, desenvolvendo-se um sistema fechado, o que permitiu a determinação da CRF pela técnica de diluição do gás hélio. Outros sistemas foram criados, ressaltando o do selo móvel e o do fole. Na década de 1970, os estudos relativos à curva de fluxo/volume foram incrementados e, com o auxílio do pneumotacógrafo desenvolvido por Fleisch, em 1925, foi possível obter o registro dos volumes deslocados a partir da integração eletrônica do sinal do fluxo ${ }^{36}$. Assim, as técnicas e os equipamentos evoluíram substancialmente. Surgiram os laboratórios de função pulmonar, e protocolos foram criados no sentido de definir normas e padrões de normalidade. Atualmente, para aferir um teste espirográfico como normal, faz-se necessário compará-lo com os valores previstos. As variáveis espirográficas apresentam valores diferentes para cada indivíduo. $\mathrm{O}$ valor esperado de cada parâmetro espirométrico é obtido mediante a equação matemática que segue o seguinte modelo: $\mathrm{X}=(\mathrm{a} \times$ idade $)$ + (b x altura) $\pm \mathrm{K}$; na qual $\mathrm{X}$ é a variável espirométrica (CVF, VEF1, etc.); a, é fator ligado à idade; $b$, fator ligado à altura; e $\mathrm{K}$, fator numérico. Para se obter os coeficientes $\mathrm{a}$, b e o fator $\mathrm{K}$, é necessário realizar espirografias em um grande número de indivíduos, de forma que todas as faixas etárias e altura, de ambos os sexos, estejam representadas. Inúmeros levantamentos dessa natureza foram realizados, existindo diversas equações de regressão aceitáveis ${ }^{33,57}$, as quais são desenvolvidas em programas específicos de informática e acopladas nos espirômetros.

\section{Aspectos históricos da gasometria}

A descoberta da presença dos gases sanguíneos na circulação data de 1670, quando o físico inglês, Robert Boyle, em 1670, (citado por Breathnach, 1972) 7 , retirou ar do sangue por meio de um dispositivo, o qual chamou bomba a vácuo. Mais tarde, Lavoisier (1777), fundador da química moderna, descreveu a parte "eminentemente respirável" do ar e a chamou de oxigênio (1779). Os primeiros estudos consistentes sobre gases sanguíneos foram realizados em 1837, pelo físico alemão, Gustav Magnus, em 1845, (citado por Breathnach, 1972) ${ }^{7}$. Este pesquisador dosou, pela primeira vez, o $\mathrm{O} 2$ e o $\mathrm{CO} 2$ no sangue arterial e venoso, comprovando, assim, que a utilização de $\mathrm{O} 2$ e a eliminação de $\mathrm{CO} 2$ se passam na intimidade dos tecidos. Com a utilização de um equipamento desenvolvido por ele próprio, que recebeu o nome de bomba de mercúrio, o cientista detectou, em si mesmo,16,6 mL de CO2 em 66,8 $\mathrm{mL}$ de sangue venoso, contestando, então, Lavoisier, ao relatar que o ar respirado não é composto somente de $\mathrm{O} 2$. Magnus ainda descreveu a maneira pela qual o $\mathrm{CO} 2$ passa pelo sangue venoso, livre de hidrogênio. Essa pesquisa foi realizada usando seu invento, a bomba de mercúrio, e com amostra de sangue coletada da jugular de cavalos e de "cidadãos que por uma pequena soma permitiram que se retirassem sangue deles mesmos". Décadas mais tarde foi determinado um outro método para medir o conteúdo de $\mathrm{O} 2$ e CO2 no plasma. É o método manométrico de Van Slike ${ }^{76}$, em que a amostra de sangue é colocada em uma câmara de vidro e depois de hemolisada, os gases são extraídos da mistura por meio de vácuo, criado manualmente. Em seguida, os gases são absorvidos com reagentes apropriados, e as determinações tensionais vão proporcionando os dados para os cálculos do conteúdo. A determinação bem executada é precisa (margem de erro de 0,03). Hoje, essa técnica está praticamente abandonada, porém foi a mais utilizada antes do advento das pressões parciais. A partir de então, vários pesquisadores contribuíram nessa linha de pesquisa e, baseados nas descobertas e fundamentos deixados por seus antecessores, Kramer e Matthes (a partir de 1934) começaram a desenvolver instrumentos (princípios da espectrofotometria) para a medida contínua da saturação de O2. Era o início dos estudos sobre oximetria de pulso, que 
culminou, alguns anos depois, com os primeiros oxímetros de larga aceitação clínica ${ }^{45,77}$. A medida dos gases, por meio de pressões parciais, nos fluidos biológicos, nesse período, passou também a ser realizada de maneira precisa e rápida, por intermédio dos métodos de cromatografia8 e polarografia $^{11}$ esta última, a mais utilizada na prática clínica atual. Esta técnica permite determinar a $\mathrm{PaO} 2$, a $\mathrm{PaCO} 2$ e o $\mathrm{pH}$ sanguíneo, por meio de eletrodos especiais. $\mathrm{O}$ da $\mathrm{PaO} 2$ é um eletrodo polarográfico, composto de um catódio de platina e de um anódio de prata (cloreto de prata), colocados em uma solução eletrolítica atrás de uma membrana plástica, por onde difunde o $\mathrm{O} 2$, para gerar corrente de platina.

\section{Aspectos históricos de procedimentos cirúrgicos e função pulmonar}

Procedimento cirúrgico e disfunção pulmonar pósoperatória é tema conhecido e pesquisado pela comunidade científica mundial de longa data. Nos primórdios do século passado, Pasteur (1910) ${ }^{53}$ relatou colapso de lobo pulmonar após operação abdominal e apontou para a dificuldade inspiratória, como causa central dessa complicação. Outros autores ${ }^{25}$ identificaram hipoxemia, em pós-operatório, secundária à respiração superficial. Em pesquisa de $1927^{26}$, o investigador correlacionou o local da incisão cirúrgica com a evolução pós-operatória da capacidade vital. As conclusões foram: a) ocorreu redução da capacidade vital pós-operatória de 5 a $80 \%$ dos valores pré-operatórios, com retorno aos valores iniciais entre um e mais de 18 dias; b) depois de operações que não envolveram incisão dos músculos respiratórios, a queda da capacidade vital é menos importante e sua recuperação, mais rápida; c) procedimentos abdominais, com maior incisão e mais próximas do diafragma, produzem as mais intensas alterações ventilatórias; d) o tamanho da incisão, bem como sua localização, são fatores mais importantes que a manipulação intra-peritoneal, no que diz respeito às alterações ventilatórias; e) operações abdominais promovem mais alterações respiratórias do que procedimentos em parede do tórax; f) as alterações ventilatórias independem do tipo de anestesia, se local ou geral; e g) as variações na capacidade vital refletem as alterações ventilatórias. Um extenso estudo58 também atestou intensa redução da capacidade vital, pós-operação abdominal, com gradual recuperação, e que a diminuição dessa variável respiratória precede as complicações pulmonares. Algumas de suas conclusões foram: procedimento cirúrgico cervical, de extremidade e de cólon retal não promovem alterações na capacidade vital; a maior repercussão sobre esta variável ocorre nas operações de abdome superior; e que a anestesia geral não tem efeito sobre a capacidade vital pós-operatória. Outro estudo 48 registrou redução de $75 \%$ da expansão torácica após operação de abdome superior. Por meio de fluoroscopia, foi mostrado que o diafragma se eleva e sua incursão diminui em $50 \%$ no pós-operatório de procedimentos abdominais. A capacidade vital se reduz em $64 \%$ e que a morfina subcutânea, pelo alívio da dor, reduz o impacto sobre esse parâmetro respiratório. Duas importantes publicações, em $1933^{4,5}$, informaram que a laparotomia afeta, profunda- mente, a respiração, produzindo: redução do volume corrente, aumento da frequência respiratória, leves alterações ventilatórias, respiração superficial, reduções significativas na capacidade vital, na CRF, na capacidade inspiratória e no volume de reserva expiratório, especialmente, após operações de abdome superior. O pesquisador aponta para a existência de outras causas indefinidas relacionadas às alterações respiratórias, pós-laparotomia. Ele ainda mostrou que após este tipo de procedimento operatório, existia marcada redução dos volumes pulmonares, com colapso pulmonar parcial, e que as alterações respiratórias mecânicas, que precediam a mencionada complicação, relacionavam-se à redução da capacidade vital. Estudos no decênio de 1950 e $1960^{23,24,74}$ apontaram os distúrbios da relação ventilaçãoperfusão como a causa principal de hipoxemia, depois de operações abdominais e torácicas e ainda, que analgesia peridural contínua é efetiva para reduzir a incidência de colapso pulmonar em pós-operatório de abdome superior $^{65}$. Outros autores ${ }^{47}$ demonstraram, após procedimento anestésico-cirúrgico, hipoxemia franca, com valor de $\mathrm{PaO} 2$ atingindo $50 \mathrm{mmHg}$ e embora resíduos de agentes anestésicos possam contribuir para hipoxemia pós-operatória, ela ocorre independente da técnica anestésica, especialmente, em operações de abdome superior ${ }^{41}$. Mostraram ainda que em procedimentos curtos (menores que 20 minutos) sua ocorrência não tem significado. Outros pesquisadores ${ }^{13}$ relataram que após procedimentos cirúrgicos abdominais (andar superior), mesmo existindo na mistura venosa $100 \%$ de O2, isto não foi suficiente para reduzir o grau de shunt. Os autores concluíram que o colapso pulmonar foi o fator predominante. Em 1969 um importante estudo ${ }^{54}$ avaliou a função pulmonar pós-operatória de diferentes tipos de operações abdominais e torácicas e concluiu que a localização da incisão é fator determinante na gênese do distúrbio ventilatório, que por sua vez, é composto somente de componentes restritivos. Em $19711^{69}$ constatouse redução da capacidade vital em até $50 \%$ dos valores pré-operatórios e hipoxemia menos evidente, em pacientes que se submeteram a operações abdominais e receberam bloqueio anestésico peridural torácico. Algumas pesquisas ${ }^{70}$ demonstraram associação importante entre a queda da $\mathrm{PaO} 2$ e a redução da CRF e do relacionamento desta última com a capacidade de fechamento que é a soma do volume residual e do volume de fechamento. A CRF diminui nas operações de abdome superior, aproximando-se da capacidade de fechamento. Essa é a principal causa de hipoxemia tardia pós-operações de abdome superior, seguida da presença de atelectasia, achado pós-operatório comum nesses tipos de procedimentos cirúrgicos e correlacionado com reduções de variáveis espirométricas ${ }^{38}$. Em $1974^{2}$, foi constatado que para a maioria dos procedimentos cirúrgicos abdominais os desarranjos ventilatórios atingem seu pico no primeiro dia de pós-operatório e, no segundo, para as toracotomias sem ressecção pulmonar. Em $1975^{43}$ foram medidas a capacidade vital, o VEF1, o volume residual e a CRF (técnica de diluição do gás hélio), antes e durante cinco dias após operações de abdome superior. Todos estes parâmetros espirométricos diminuíram no pós-operatório, com redução 
máxima no primeiro e segundo dias. Enfraquecimento da função ciliar, com prejuízo no transporte de muco, é considerado uma causa de atelectasia e consequente redução na $\mathrm{PaO} 2$, especialmente, em pacientes submetidos a procedimentos cirúrgicos de abdome superior e torácico. Em 1975 foi realizada pesquisa inédita. Os autore ${ }^{21}$ observaram 25 pacientes, sete dos quais submetidos a procedimentos ortopédicos de extremidades e, 18 , a procedimentos vasculares intra-abdominais. Os pacientes foram submetidos à anestesia geral, com entubação traqueal e, no final do ato cirúrgico insuflava-se pó de tântalo (metal para fabricação de arames, tubulações, etc.) em ambos os pulmões. Esse procedimento foi controlado por fluoroscopia e realizado utilizando um catéter introduzido por dentro da cânula traqueal, que recebia o elemento metálico em pó,

proveniente de um injetor com ar comprimido. Em seguida, os pacientes eram extubados. Posteriormente realizaram-se radiografias de tórax, imediatamente após a insuflação, como também, depois de seis, 18, 26 e 48 horas e, depois disso, quando necessário. Nos pacientes que se submeteram a procedimentos ortopédicos, o clearance do tântalo foi progressivo e, usualmente, completava-se em 48 horas. Vale salientar que atelectasia não ocorreu nesse grupo. Dos outros 18 enfermos, 14 apresentaram clearance marcadamente anormal, que se completava acima de seis dias. Por outro lado, em 16 pacientes foi detectada a coleção muco-tântalo, no brônquio correspondente. A coleção precedia e sempre foi acompanhada, radiograficamente, de atelectasia visível em 14 pacientes (em seis, atelectasia lobar e, em oito, segmentar). Assim, conclui-se que a atividade muco-ciliar prejudicada está associada à atelectasia pós-operatória. Outros autores ${ }^{52}$ acompanharam os dois primeiros dias de pós-operatório de pacientes submetidos a diversos tipos de procedimentos cirúrgicos e constataram que hipoxemia foi um achado constante em todos os enfermos que se submeteram à anestesia geral e que a redução da $\mathrm{PaO} 2$ sinaliza para atelectasia pós-operatória. Os efeitos de operações de abdome superior no diafragma foram observados por alguns pesquisadores ${ }^{67}$. Constataram-se marcada disfunção diafragmática, que provavelmente, é a principal causa de padrão espirométrico restritivo durante a convalescença das operações. O retorno deste músculo ao seu estado normofuncional ocorre no período de sete ou mais dias, e o alívio da dor pós-operatória não acelera este retorno, contudo outros autores relatam que analgesia eficiente melhora a função do diafragma ${ }^{32}$. Ainda em relação à dor pós-operatória alguns estudos apontam que o alívio da dor nos procedimentos cirúrgicos de abdome superior melhora e ameniza os distúrbios ventilatórios pós-operatórios, reduzindo, inclusive, a morbidade ${ }^{37,63,66,80}$. Por outro lado, há estudos que relatam que o alívio da dor pós-operatória não melhora a função pulmonar ${ }^{6,29,30}$. Outros autores ${ }^{16}$, também pesquisaram sobre disfunção diafragmática após operações de abdome superior, verificando a contratilidade deste músculo. O nervo frênico, bilateralmente, foi estimulado eletricamente para obtenção da função contrátil pós-operatória do diafragma. Os autores concluíram que a função contrátil do diafragma não é alterada no pós-operatório de procedimentos cirúrgicos de abdome superior e que a disfunção do músculo é secundária a outros mecanismos, assim como possíveis reflexos periféricos, os quais podem inibir a função do nervo frênico. Mais tarde ${ }^{15,71}$, relacionado ao enfraquecimento do músculo diafragma, foi observado que a disfunção diafragmática é a principal responsável pela gênese dos distúrbios ventilatórios após operações de abdome superior. Isso ocorre devido à inibição reflexa do nervo frênico. A via reflexa aferente é originária, provavelmente, do plexo celíaco simpático ou de outro gânglio simpático abdominal superior. Esse mesmo mecanismo explica a disfunção restritiva que também ocorre nos procedimentos laparoscópicos ${ }^{17}$. A região da colecistectomia laparoscópica envolve vias viscerais aferentes da região mesentérica, que são áreas reflexógenas, promotoras de alteração e enfraquecimento da função diafragmática ${ }^{34,59}$. Outros estudiosos ${ }^{18}$, mencionaram a ocorrência de redução da função pulmonar (CRF e PaO2), depois de operações de abdome superior e torácica. Essas alterações predispõem complicações pulmonares pós-operatórias. Alguns autores relataram complicações pulmonares relacionadas a maior tempo cirúrgico. Assim, operações com menos de uma hora, entre uma e duas, entre duas e quatro, e mais que quatro horas, apresentam, respectivamente, 4, 23, $38 \mathrm{e}$ $73 \%$ de complicações pulmonares. Igualmente, anestesia geral, com mais de duas horas de intubação traqueal, tende a se relacionar com maior complicação pós-operatória ${ }^{35,78}$. Outros autores ${ }^{50}$, considerando a disfunção diafragmática, pesquisaram a atividade desse músculo em pacientes que receberam bloqueio anestésico peridural torácico com bupivacaína $0,5 \%$, e se submeteram à operação de aorta abdominal (abdome superior). Foi demonstrado que o bloqueio anestésico peridural torácico produz aumento na atividade diafragmática pós-operatória e, que a hipótese mais atraente para explicar as consequências do prejuízo da disfunção pós-operatória deste músculo, nesses procedimentos, é a interrupção de vias reflexas aferentes. Alguns autores ${ }^{40}, 1996$, não verificaram diferenças entre um grupo de enfermos operados de colecistectomias laparoscópicas e outro submetido à técnica aberta com mini-laparotomia, no que diz respeito à permanência hospitalar ou recuperação pós-operatória. Além disso, os autores consideraram que o tempo operatório do procedimento laparoscópico foi muito maior do que o procedimento de pequena incisão. No final da década de 1980, com o advento das operações laparoscópicas, foram apresentadas as primeiras publicações científicas relativas à esse técnica operatória. Dessa época até o decênio 2000, várias pesquisas científicas referiram a superioridade das laparoscopias sobre os procedimentos abertos (por laparotomia) relacionados a uma série de parâmetros: a) distúrbios ventilatórios restritivos de menor monta, com queda menos acentuada das variáveis espirométricas CVF, VEF1 e CRF ${ }^{19,31}$; b) recuperação mais rápida da função pulmonar no que diz respeito à espirometria ${ }^{61}$; c) mínimas alterações sobre a $\mathrm{PaO}^{60}$; d) redução de complicações pulmonares ${ }^{3}$; e) menor disfunção diafragmática ${ }^{12}$ e f) dor pós-operatória de menor intensidade ${ }^{42}$. Um outro estudo $^{49}$ comparou no pré, primeiro, terceiro e quinto dias 
de pós-operatório de gastroplastia (abdome superior) de obesos mórbidos, as variáveis espirométricas volumeminuto, volume-corrente e capacidade vital. Os autores concluíram que, para este grupo, ocorreu disfunção ventilatória restritiva equivalente aos observados em não-obesos.

\section{CONCLUSÕES}

A espirometria e a $\mathrm{PaO} 2$ arterial são importantes aferidores da função pulmonar após operações de abdome superior. Esses procedimentos cirúrgicos, especialmente os maiores, invariavelmente, evoluem com distúrbios ventilatórios restritivos pós-operatórios, que tornam os pulmões mais vulneráveis e precedem as complicações pulmonares ${ }^{18}$. A fisiopatogênese desses distúrbios relaciona-se com vários fatores que se somam. Sob o ponto de vista de localização do ato operatório, sua proximidade com o diafragma, estimulando sítios cirúrgicos reflexógenos, causando disfunção deste músculo ${ }^{67}$, é o principal fator determinante destas desordens. Contudo várias causas acessórias estão envolvidas e inter-relacionam entre si: dor pós-operatória, reduzida capacidade de tossir e de eliminar secreções devido ao enfraquecimento da função mucociliar, atelectasia, duração do ato anestésico-cirúrgico, tamanho da incisão cirúrgica e agressão muscular. A técnica cirúrgica laparoscópica é superior à aberta no que se refere à função pulmonar pós-operatória e, sempre que possível, deve ser a preferida. Nas indicações de operações abertas, quando viável, utilizar de pequenas incisões (minilaparotomia) e com tempo cirúrgico abreviado, o que pode diminuir estímulos reflexógenos e amenizar a disfunção diafragmática. Embora exista controversias em relação ao tratamento da dor pós-operatória na melhora da função pulmonar, a impressão clínica que se tem é que se trata de variável relevante e piora a disfunção diafragmática ${ }^{32,46}$. Assim, terapêutica eficiente da crise álgica pode amenizar os distúrbios ventilatórios restritivos, aliviando a dor e melhorando a função diafragmática. Nesse contexto, a presença de distúrbios ventilatórios restritivos e hipoxemia pós-operatória é intercorrência esperada em operações de médio e grande portes de abdome superior e, que precede complicações clínicas pulmonares. Assim, nos pacientes selecionados para operações abdominais, variáveis tais como técnica laparoscópica, mini-laparotomia, analgesia pós-operatória, reduzido tempo cirúrgico e de entubação traqueal podem reduzir complicações pulmonares, que vão desde uma simples infecção, até insuficiência respiratória e morte, principalmente em pneumopatas prévios.

Ramos GC, Pereira E, Gabriel-Neto S, Oliveira EC. Historical aspects of arterial oxygen pressure and spirometry related to abdominal operations. ABCD Arq Bras Cir Dig 2009;22(1):50-6

ABSTRACT - Introduction - Operations, particularly those of the upper abdomen, can be accompanied by hypoxemia and restrictive ventilatory disturbances. The objectives of the present review were: a) to provide a retrospective of spirometric techniques and laboratory assessment of hypoxemia by means of arterial oxygen pressure; b) to review the main factors responsible for alterations in postoperative pulmonary function. Methods - A historical overview is given of the main aspects of spirometry and $\mathrm{PaO} 2$, measurement, considering these exams as measures of pulmonary function after abdominal operations. Conclusion - Operations on the upper part of the abdomen may be followed by hypoxemia and restrictive ventilatory disturbances, whose principal cause is diaphragmatic dysfunction which can be minimized by means of laparoscopy and effective post-operative pain treatment.

HEADINGS - Abdominal surgery. Post-operative. Pulmonary function.

\section{REFERÊNCIAS}

1 Aboussouan LS, Stoller JK - Perioperative pulmonary care, in: Cherniak, NS, Altose MD, Homma I - Rehabilitation of patient with respiratory disease. New York: The Mc Graw - Hill, 1999. p. 561-575.

2 Ali J, Weisel RD, Layug AB et al -Consequences of postoperative alterations in respiratory mechanics. Am J Surg, 1974; 128: 376 - 382.

3 Barnett RB, Clement GS, Drizin GS et al - Pulmonary changes after laparoscopic cholecystectomy. Surg Laparosc Endosc, 1992; 2: 125 - 127.

4 Beecher HK - The measured effect of laparotomy on the respiration. J Clin Invest, 1933; 12: 639 - 650.

5 Beecher HK - Effect of laparotomy on lung volume. Demonstration of a new type of pulmonary collapse. J Clin Invest, 1933; 12: 651 - 658.

6 Bonnet F, Blery C, Zatan M et al - Effect of epidural morphine on post-operative pulmonary dysfunction. Acta Anaesthesiol Scand, 1984; 28: 147 - 151.

7 Breathnach CS - The development of blood gas analysis. Med Hist, 1972; 16 : $51-62$.

8 Brenner N, Cispunski E - Gas chromatographic analysis of mixtures containing oxygen, nitrogen and carbon dioxide. Ann N Y Acad Sci, 1959; 72: 705 - 713.

9 Burrows B, Earle RH - Prediction of survival in patients with chronic airway obstruction. Am Rev Respir Dis, 1969; 99: 865 - 871.

10 Christie RV - The lung volume and its subdivisions: I. Methods of measurement. J Clin Invest, 1932; 11: 1099 -1118.

11 Clark LC Jr, Wolf R, Granger D et al - Continuous recording of blood oxygen tensions by polarography. J Appl Physiol, 1953; 6: 189 -193.

12 Couture JG, Chartrand D, Gagner M et al - Diaphragmatic and abdominal muscle activity after endoscopic cholecystectomy. Anesth Analg, 1994; 78: $733-739$.
13 Diament ML, Palmer KN - Venous/arterial pulmonary shunting as the principal cause of postoperative hypoxaemia. Lancet, 1967; 1: 15 - 17.

14 Dias RM, Chauvet PR, Siqueira, HR et al - Testes de função respiratória: do laboratório à aplicação clínica com 100 exercícios para diagnóstico. São Paulo: Atheneu, 2000. p. 5 - 9.

15 Dureuil B, Cantineau JP, Desmonts JM - Effects of upper or lower abdominal surgery on diaphragmatic function. Br J Anaesth, 1987; 59: 1230 - 1235.

16 Dureuil B, Viire N, Cantineau JP et al - Diaphragmatic contractility after upper abdominal surgery. J Appl Physiol, 1986; 61: 1775 - 1780.

17 Erice F, Fox GS, Salib Y M et al - Diaphragmatic function before and after laparoscopic cholecystectomy. Anesthesiology, 1993; 79: 966 - 975.

18 Fairshter RD, Williams JH Jr - Pulmonary physiology in the postoperative period. Crit Care Clin, 1987; 3: 287 - 306.

19 Frazee RC, Roberts JW, Symmonds RE et al - Open versus laparoscopic cholecystectomy. A comparison of postoperative pulmonary function. Ann Surg, 1991; $213: 651-653$.

20 Gaensler EA - Analysis of ventilatory defect by timed capacity measurements. Am Rev Tuberc, 1951; 64: 256 - 278.

21 Gamsu G, Singer MM, Vincent HH et al - Postoperative impairment of mucous transport in the lung. Am Rev Respir Dis, 1976; 114: 673 - 679.

22 Gardner RM, Crapo RO, Nelson SB - Spirometry and flow-volume curves. Clin Chest Med, 1989; 10: 145 - 154.

23 Georg J, Hornum I, Mellemgaard K - The mechanism of hypoxaemia after laparotomy. Thorax, 1967; 22: 382 - 326. 
24 Gordh T, Linderholm H, Norlander O. Pulmonary function in relation to anesthesia and surgery evaluated by analysis of oxygen tension of arterial blood. Acta Anaesthesiol Scand, 1958; 2: 15 - 26.

25 Haldane JS, Meakins JL, Priestley JG - The effect of shallow breathing. J Physiol, 1919; 52: 433 - 453.

26 Head JR - The effect of operation upon the vital capacity. Bost Med Surg J, 1927; 197: $83-87$.

27 Hutchinson J - On the capacity of the lungs, and on the respiratory movements, with the view of establishing a precise and easy method of detecting disease by the spirometer. Lancet, 1846; 1: 630 - 632.

28 Hyatt RE, Schilder DP, Fry DL - Relationship between maximum expiratory flow and degree of lung inflation. J Appl Physiol, 1958; 13: 331 - 336.

29 Jayr C, Mollie A, Bourgain JL et al - Postoperative pulmonary complications: general anesthesia with postoperative parenteral morphine compared with epidural analgesia. Surgery, 1988; 104: 57 - 63.

30 Jayr C, Thomas H, Rey A et al - Postoperative pulmonary complications. Epidural analgesia using bupivacaine and opioids versus parenteral opioids. Anesthesiology, 1993; 78: 666 - 676.

31 Karayiannakis AJ, Makri GG, Mantzioka A et al - Postoperative pulmonary function after laparoscopic and open cholecystectomy. Br J Anaesth, 1996; 77: 448-52.

32 Kiya T, Fujimura N, Okanuma $M$ et al - Effect of continuous extradural infusion of bupivacaine on diaphragmatic function after upper abdominal surgery. Masui, 2003; 52: 500 - 504 .

33 Knudson RJ, Lebowitz MD, Holberg CJ et al - Changes in the normal maximal expiratory flow-volume curve with growth and aging. Am Rev Respir Dis, 1983; 127: 725 - 734 .

34 Kostreva DR, Hopp F., Zuperku EJ et al - Respiratory inhibition with sympathetic afferent stimulation in the canine and primate. J Appl Physiol, 1978; 44: 718 - 724.

35 Kroenke K, Lawrence VA, Theroux JF et al - Operative risk in patients with severe obstructive pulmonary disease. Arch Intern Med, 1992; 152: 967 - 971.

36 Lemle A - Provas de função pulmonar na prática diária. Rio de Janeiro: Publicações Científicas, 1994. p. 29 - 44.

37 Lewis KS, Whipple JK, Michael KA et al - Effect of analgesic treatment on the physiological consequences of acute pain. Am J Hosp Pharm, 1994; 51: 1539 - 1554.

38 Lindberg P, Gunnarsson L, Tokics L et al - Atelectasis and lung function in the postoperative period. Acta Anaesthesiol Scand, 1992; 36: 546 - 553.

39 Lundsgaard C, Schierbeck K - Studies on lung volume IV. Investigations on admixture of air in the lungs with other air. Acta med Scandinav, 1923; 58: $470-485$.

40 Majeed AW, Troy G, Nicholl JP et al -. Randomised, prospective, single-blind comparison of laparoscopic versus small-incision cholecystectomy. Lancet, 1996; 347: 989 - 994.

41 Marshall BE, Millar RA - Some factors influencing post-operative hypoxaemia. Anaesthesia, 1965; 20: 408 - 428 .

42 Mcmahon A.J, Russell IT, Ramsay G et al - Laparoscopic and minilaparotomy cholecystectomy: a randomized trial comparing postoperative pain and pulmonary function. Surgery, 1994; 115: 533 - 539.

43 Meyers JR, Lembeck L, O'kane $\mathrm{H}$ et al - Changes in functional residual capacity of the lung after operation. Arch Surg, 1975; 110: 576 - 583.

44 Mortimer KM, Fallot A, Balmes JR et al - Evaluating the use of a portable spirometer in a study of pediatric asthma. Chest, 2003; 123: 1899 - 1907.

45 Nahas GG - Spectrophotometric determination of haemoglobin and oxygen in whole hemolysed blood. Science, 1951; 113: 723 - 25.

46 Nguyen NT, Lee SL, Goldman C et al - Comparison of pulmonary function and postoperative pain after laparoscopic versus open gastric bypass: a randomized trial. J Am Coll Surg, 2001; 192: 469 - 476.

47 Nunn JF, Payne JP - Hypoxaemia after general anaesthesia. Lancet, 1962; 29: $631-632$.

48 Overholt R - Post-operative pulmonary hypoventilation. JAMA, 1930; 95: $1484-1487$

49 Paisani DM, Chiavegato LD, Faresin SM - Volumes, capacidades pulmonares e força muscular respiratória no pós-operatório de gastroplastia. J Bras Pneumol, 2005; 31: 125-132

50 Pansard JL, Mankikian B, Bertrand M et al - Effects of thoracic extradural block on diaphragmatic electrical activity and contractility after upper abdominal surgery. Anesthesiology, 1993; 78: 63 - 71.

51 Pappenheimer J - Standardization of definitions and symbols in respiratory physiology. Fed Proc, 1950; 9: 602 - 605.

52 Parfrey PS, Harte PJ, Quinlan JP at al - Pulmonary function in the early postoperative period. Br J Surg, 1977; 64: 384 -389.
53 Pasteur W - Active lobar collapse of the lung after abdominal operation: A contribution to the study of post operative lung complications. Lancet, 1910; 2: $1080-1083$.

54 Pecora DV - Predictability of effects of abdominal and thoracic surgery upon pulmonary function. Ann Surg, 1969; 170: $101-108$.

55 Pereira AC - Aplicações da espirometria. In: Sociedade Brasileira de Pneumologia e Tisiologia. Diretrizes para testes de função pulmonar 2002. J Pneumol, 2002; 28: 68 - 80

56 Pereira CAC - Técnica. In: Sociedade Brasileira de Pneumologia e Tisiologia. Diretrizes para testes de função pulmonar 2002. J Pneumol, 2002; 28: 2 - 3.

57 Pereira CAC, Barreto SP, Simões JG et al - Valores de referência para espirometria em uma amostra da população brasileira adulta. J Pneumol, 1992; 18: $10-22$.

58 Powers JH - Vital capacity. Its significance in relation to postoperative pulmonary complications. Arch Surg, 1928; 17: 304 - 323.

59 Prabhakar NR, Marek W, Loeschcke HH - Altered breathing pattern elicited by stimulation of abdominal visceral afferents. J Appl Physiol, 1985; 58: 1755 -1760 .

60 Ramos GC, Pereira E, Neto SG et al - Influencia da morfina peridural na funcão pulmonar de pacientes submetidos a colecistectomia aberta. Rev Bras Anestesiol, 2007; 57: 366 - 381.

61 Ravimohan SM, Kaman L, Jindal R et al - Postoperative pulmonary function in laparoscopic versus open cholecystectomy: prospective, comparative study. Indian J Gastroenterol, 2005; 24: 5 - 8.

62 Ruppel GL - Manual of pulmonary function testing. 7.ed. St. Louis: Mosby, 1994. p. $237-342$.

63 Sakura S, Yanagidani T, Saito Y et al - Changes in pulmonary function during continuous epidural bupivacaine with or without morphine following upper abdominal surgery. J Anesth, 1990; 4: 319 - 326.

64 Silva LCC, Rubin AS, Silva LMC et al - Espirometria na prática médica. Revista AMRIGS, 2005; 49: 183 - 194.

65 Simpson BR, Parkhouse J, Marshall R et al - Extradural analgesia and the prevention of postoperative respiratory complications. Br J Anaesth, 1961; 33: $628-641$.

66 Simpson T, Wahl G, Detraglia M et al - A pilot study of pain, analgesia use, and pulmonary function after colectomy with or without a preoperative bolus of epidural morphine. Heart Lung, 1993; 22: 316 - 327.

67 Simonneau G, Vivien A, Sartene R et al - Diaphragm dysfunction induced by upper abdominal surgery. Role of postoperative pain. Am Rev Respir Dis, 1983; 128: 899 - 903.

68 Sociedade Brasileira de Pneumologia e Tisiologia. I Consenso brasileiro sobre espirometria. J Pneumol, 1996; 22: 122 - 129.

69 Spence AA, Smith G - Postoperative analgesia and lung function: a comparison of morphine with extradural block. Br J Anaesth, 1971; 43: 144 - 148.

70 Spence AA, Alexander JL - Mechanisms of postoperative hipoxaemia. Proc R Soc Med, 1972; 65: 12 - 14

71 Sprung J, Cheng EY, Nimphius N et al - Diaphragm dysfunction and respiratory insufficiency after upper abdominal surgery. Plucne Bolesti, 1991; 43: 5 - 12.

72 Tavares FMB, Silva L.CC, Rubin AS - O volume expiratório forçado no primeiro segundo não é suficiente para avaliar resposta broncodilatadora em doença pulmonar obstrutiva crônica. J Bras Pneumol, 2005; 31: 407 - 414

73 Tiffeneau R, Pinelli A - Air circulante et air captif dans l'exploration de la fonction ventilatrice pulmonaire. Paris Med, 1947; 133: 624 - 628.

74 Troell L - Post-operative changes in circulation and the effects of oxygen therapy. Acta Chir Scand, 1951; 102: 203 - 214.

75 Van Slyke DD, Binger CL - The determination of lung volume without forced breathing. J Exper Med, 1923; 37: 457 - 470.

76 Van Slyke DD, Neill JM - The determination of gases in blood and other solutions by vacuum extraction and manometric measurement. I. J Biol Chem, 1924; 61: 523 - 573

77 Wade OL, Bishop JJ, Cumming G et al - Method for the rapid estimation of percentage oxygen saturation and oxygen content in blood. Brit med J, 1953; 24: 902 - 907.

78 Wong DH, Weber EC, Schell MJ et al - Factors associated with postoperative pulmonary complications in patients with severe chronic obstructive pulmonary disease. Anesth Analg, 1995; 80: 276 - 284.

79 Yernault JC, Pride N, Laszlo G - How the measurement of residual volume developed after Davy (1800). Eur Respir J, 2000; 16: 561 - 564.

80 Zenz M, Piepenbrock S, Tryba M et al - Peridural opiate analgesia. Clinical results of a 2-year study. Anaesthesist, 1983; 32: 289 - 294.

Fonte de financiamento: não há Conflito de interesse: não há Recebido para publicação: 19/07/2008 Aceito para publicação: 14/12/2008 